\title{
3D AMR simulations of the evolution of the diffuse gas cloud G2 in the Galactic Centre
}

\author{
M. Schartmann ${ }^{1,2,3}$, A. Ballone ${ }^{1,2}$, A. Burkert ${ }^{1,2,4}$, S. Gillessen ${ }^{2}$, \\ R. Genzel ${ }^{2}$, O. Pfuhl ${ }^{2}$, F. Eisenhauer ${ }^{2}$, P.M. Plewa ${ }^{2}$, T. Ott ${ }^{2}$, \\ E.M. George ${ }^{2}$ and M. Habibi ${ }^{2}$ \\ ${ }^{1}$ University Observatory Munich, Scheinerstraße 1, D-81679 München, Germany \\ ${ }^{2}$ Max-Planck-Institute for extraterrestrial Physics, Postfach 1312, Giessenbachstr., \\ D-85741 Garching, Germany \\ ${ }^{3}$ Centre for Astrophysics and Supercomputing, Swinburne University of Technology, \\ P.O. Box 218, Hawthorn, Victoria 3122, Australia \\ email: mschartmann@swin.edu .au \\ ${ }^{4}$ Max-Planck-Fellow
}

\begin{abstract}
With the help of 3D AMR hydrodynamical simulations we aim at understanding G2's nature, recent evolution and fate in the coming years. By exploring the possible parameter space of the diffuse cloud scenario, we find that a starting point within the disc of young stars is favoured by the observations, which may hint at G2 being the result of stellar wind interactions.
\end{abstract}

Keywords. accretion, black hole physics, Galaxy: center, hydrodynamics, ISM: clouds, ISM: evolution

\section{Observations}

Gillessen et al. (2012) discovered a fast moving object within the range of the S-star cluster close to Sgr A*. VLT NACO images show the object in L'-band, but not in $\mathrm{K}$-band, indicating that it is a dusty, ionised gas cloud. The spatially resolved ionised gas emission (especially in the Brackett- $\gamma$ line) monitored with the SINFONI instrument allows to accurately constrain the orbit around Sgr A* to be highly eccentric $(\mathrm{e}=0.98)$ with a peri-centre passage at a distance of 2400 Schwarzschild radii. The cloud has a mass of 3 earth masses and an orbital period of 400 years. The tidal interaction with the central black hole is clearly visible in the developing gradients in observed positionvelocity (PV) diagrams (Gillessen et al. 2013a, 2013b). An overlay of the PV diagrams of the years 2004 to 2014 shows that G2, a second cloud G1 and the tail might form a stream of gas (Pfuhl et al. 2015).

\section{Simulated evolution of a diffuse cloud}

We employ 3D AMR hydro-dynamical simulations using the PLUTO code in order to follow the evolution of a diffuse gas cloud which starts initially in pressure equilibrium with an ADAF-like atmosphere on the observed orbit. Fig. 1 shows that the spherical cloud is initially compressed due to the radially increasing atmospheric pressure and ram pressure interaction (Fig. 1a). Close to peri-centre, the massive black hole leads to tidal stretching (Fig. 1b) and after the peri-centre passage, the cloud develops a nozzle-like accretion stream feeding gas towards the centre (Fig. 1c) with small infall rates. However, despite the seemingly disruption of the cloud, the (normalised) Brackett- $\gamma$ emission on the sky closely follows the observed orbit, even after the cloud's peri-centre passage. 

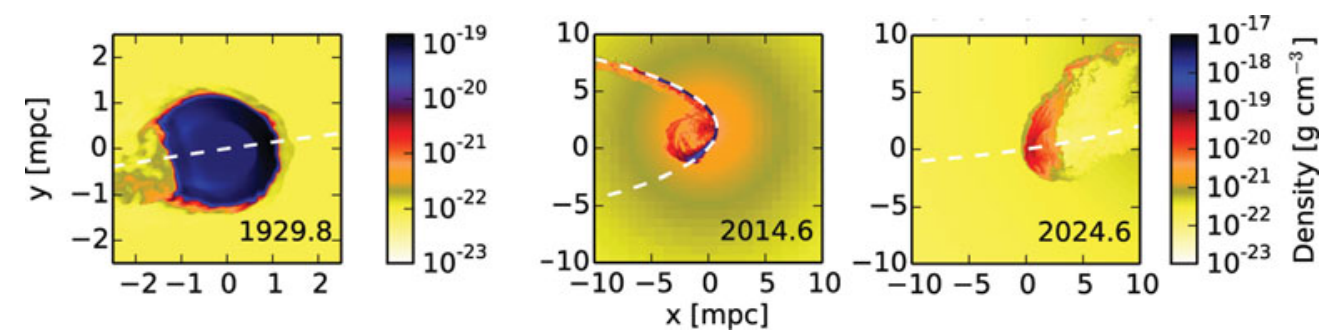

Figure 1. Density distributions showing the evolution from a spherical cloud to a thin filamentary structure: (a) compression due to increasing ambient pressure and ram pressure, (b) tidal interaction and (c) accretion towards Sgr A* in a nozzle-like accretion stream. Adapted from Schartmann et al. (2015).

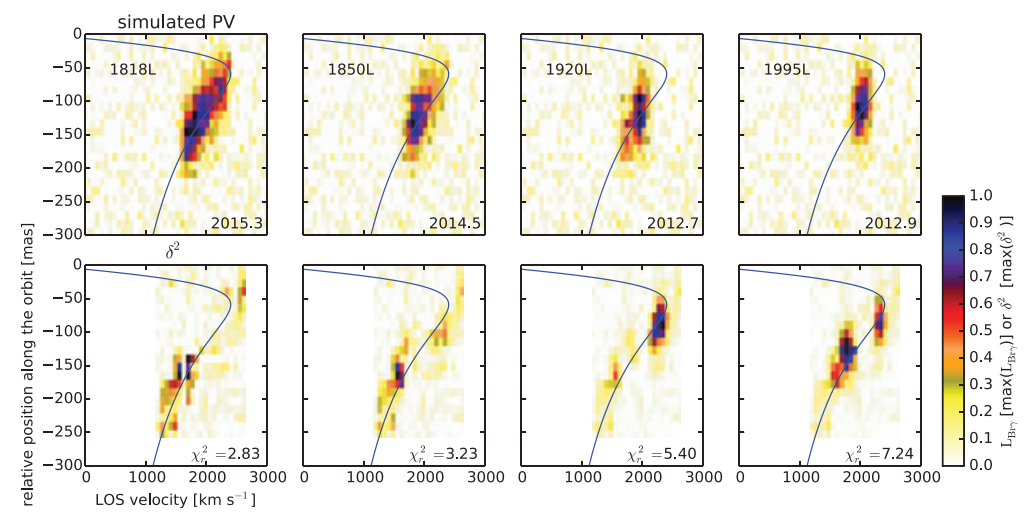

Figure 2. Simulated PV diagrams (upper row) for various starting times of the clouds (as annotated in the upper left corner) and the normalized residual arrays after directly comparing them to the 2012 observed PV diagrams (lower row). The blue curve corresponds to G2's nominal orbit. A starting point of the clouds close to peri-centre gives the best adaptation of the data. Taken from Schartmann et al. (2015).

\section{Comparison to observations and conclusions}

By directly comparing our simulated position-velocity (PV) diagrams for various starting times (in pressure equilibrium) along the orbit (upper row in Fig. 2) with the observed ones (residuals in second row in Fig. 2), we find better correspondence for models with a starting time close to apo-centre of the best-fit G2 orbit, as inferred from a reduced $\chi^{2}$ analysis. This might indicate a formation scenario due to stellar wind interaction within the disc of young stars (Calderón et al. 2016) or G2's origin from a gas streamer. Brackett- $\gamma$ light curves for these simulations show a (partly physical) mixing plateau and are roughly in agreement with the data. Similarly good overall agreement is found for the Compact Source Scenario (see conference contribution by Alessandro Ballone). However, in the latter scenario, the source leaves G2 behind in approximately 5 years from now and forms a separate cloud, allowing us to distinguish the two scenarios.

\section{References}

Calderón, D., Ballone, A., Cuadra, J., et al. 2016, MNRAS, 455, 4388

Gillessen, S., Genzel, R., Fritz, T. K., et al. 2012, Nature, 481, 51

-. 2013a, ApJ, 763, 78

-. 2013b, ApJ, 774, 44

Pfuhl, O., Gillessen, S., Eisenhauer, F., et al. 2015, ApJ, 798, 111

Schartmann, M., Ballone, A., Burkert, A., et al. 2015, ApJ, 811, 155 